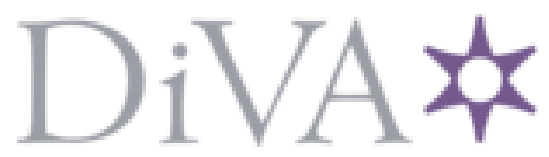

http://www.diva-portal.org

\title{
Postprint
}

This is the accepted version of a paper presented at Cyberworlds 2015,7-9 Oct 2015, Gotland, Sweden.

Citation for the original published paper:

Hayashi, M., Bachelder, S., Nakajima, M. (2015)

Open Framework Facilitating Automatic Generation of CG Animation from Web Site. In:

N.B. When citing this work, cite the original published paper.

Permanent link to this version:

http://urn.kb.se/resolve?urn=urn:nbn:se:uu:diva-268615 


\title{
Open Framework
}

\section{Facilitating Automatic Generation of CG Animation from Web Site}

\author{
Masaki Hayashi $^{\dagger}$ Steven Bachelder ${ }^{\dagger}$ Masayuki Nakajima ${ }^{\dagger *}$ \\ †Department of Game Design \\ Uppsala University, Campus Gotland \\ Visby, Sweden \\ ${ }^{\ddagger}$ Kanagawa Institute of Technology \\ Kanagawa, Japan \\ masayuki.nakajima@gmail.com \\ \{masaki.hayashi, steven.bachelder\}@speldesign.uu.se
}

\begin{abstract}
We have been studying and developing the system which enables to generate Computer Graphics Animation (CGA) automatically by processing HTML data of Web site. In this paper, we propose an open framework to facilitate this. The framework is functioning all at a server side, obtaining the HTML, converting it to a script describing the CGA story and updating the script. And at a client side, a user accesses the script on the server to visualize it by using real-time CG character with synthesized voice, camera work, superimposing, sound file playback etc. We have constructed the framework on the server and deployed the substantial engines to convert Web sites to CGAs. This paper describes the detail of the framework and also shows some example projects providing automatically generated News show, Talk show and personal Blog visualization.
\end{abstract}

Keywords-animation, text visualization, media conversion, scripting langauge

\section{INTRODUCTION}

We have been studying the technology called Text-ToVision (T2V) which is able to convert text to Computer Graphics Animation (CGA) [1]. T2V is developed based on TV program Making Language (TVML) which has been used in many ways [2]. A TVML script is interpreted by special software called TVML engine to generate CGA by using realtime CG character with synthesized voice, CG camera work, superimposing, displaying image data, playback of movie and sound data, etc. Since the text written in TVML can be converted to CGA, any type of text source is viable to be turned to CGA by analyzing the original text to obtain TVML script. Using this methodology, many attempts have been made to visualize information sources available on the Web [3-4]. However, those attempts are more or less research based and developed as standalone applications or services. We have been in lack of shared space on the Internet where any individual people enable to develop their own automatic CGA generation experiments and services.

In this time, we have designed and developed an open framework to facilitate this automatic animation creation from information sources on the Web. Our aim is to provide the development environment to individual people to make their own services of broadcasting the CGAs. In order to do this, we have made a service site on a server where the developers can make their own conversion engines and upload them to the server instantly so that people can watch the generated CGAs (i.e. News show, Talk show, Weather report, etc.). We can also say that this automatic animation generation system on the Internet could become "Auto broadcast station". Our ultimate goal is to establish the technology of constructing Internet broadcast station providing CGAs generated automatically from the Web sources [5].

This paper illustrates the architecture of the framework and describes the technical details. Also some actually implemented auto CGA generation services are introduced followed by the demonstrations.

\section{PRINCIPLE OF AUtOMATIC CGA GENERATION}

Figure 1 shows the basic principle of the automatic CGA generation from Web site. HTML data of a specific Web site is first obtained, then it is converted to an arbitrary XML script (it is called "ARC script", later explained), then it is converted to a TVML script and it is ultimately played back by a TVML engine to produce CGA. There are two conversion engines in this figure which we named SEC engine (taken from "SEmantic Conversion") and ARC engine (taken from "ARbitrary Conversion") respectively. Both engines work

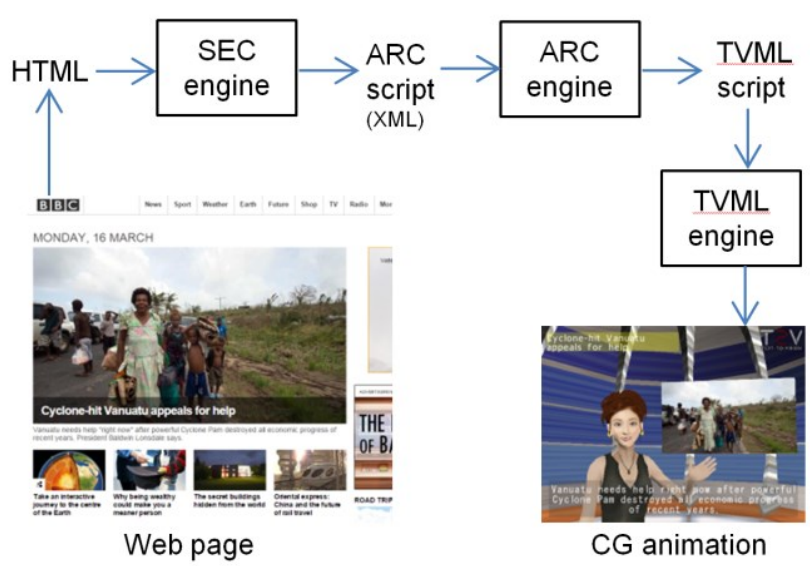

Figure 1: Automatic CGA generation from Web page 


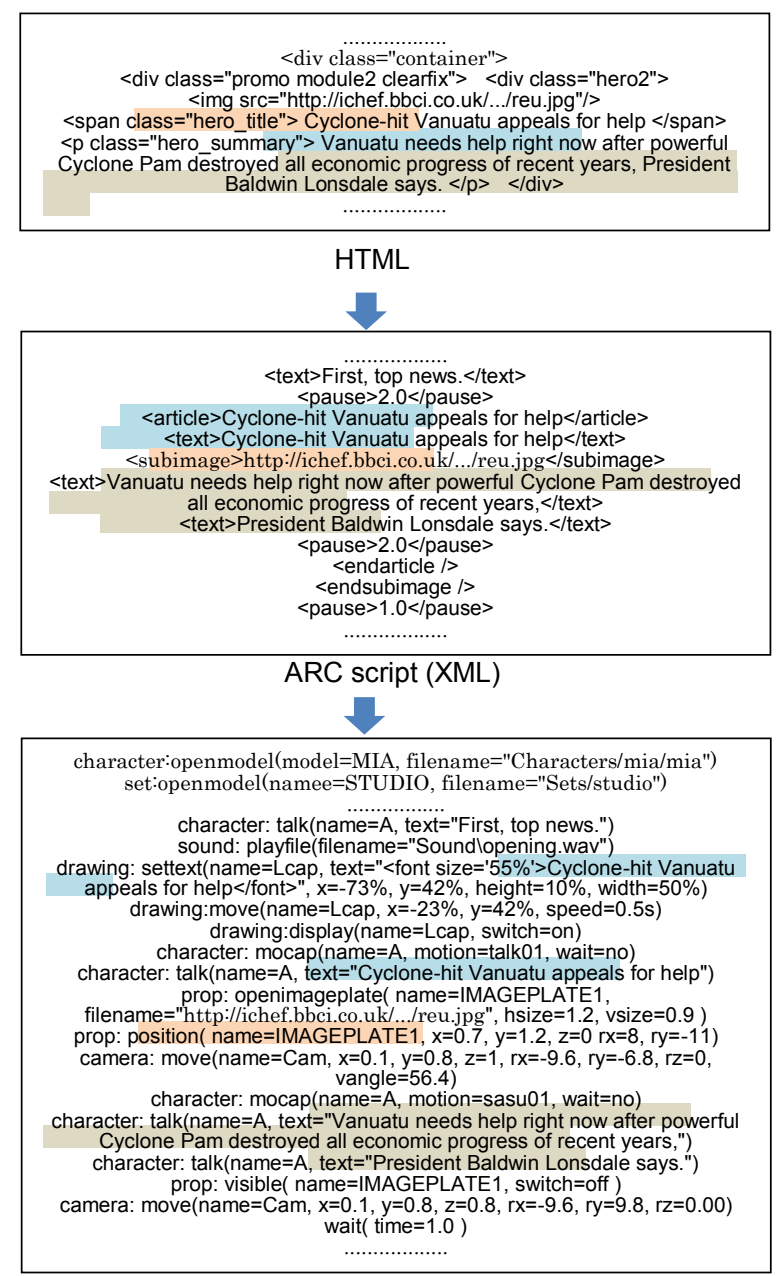

TVML script

Figure 2: Conversion from HTML to TVML via ACR script (simplified)

simply processing input text to produce output text. In our context, the SEC converts HTML to XML (ARC script) and the ARC converts XML (ARC script) to TVML.

Fugure 2 shows an example of this process when it is applied to automatic news show generation. The SEC engine is designed to extract "Title of an article", "URL of an image file in the article" and "Main text of the article" from a given HTML to output XML data (ARC script) which describes a story made by those three items using XML tags. The ARC script does not contain visual elements used in the output CGA such as what character is used, how the camera angles are going to be and so on. Those visual elements are maintained by ARC engine. The ARC engine interprets a story expressed in an ARC script to generate TVML script which describes all the visual elements used in the CGA.

As explained above, we define SEC and ARC having different roles. The former one makes a story from the source Web page and the latter one gives visual expression to the story. The benefits of this method would be:

- To enable to design two different engines separately.

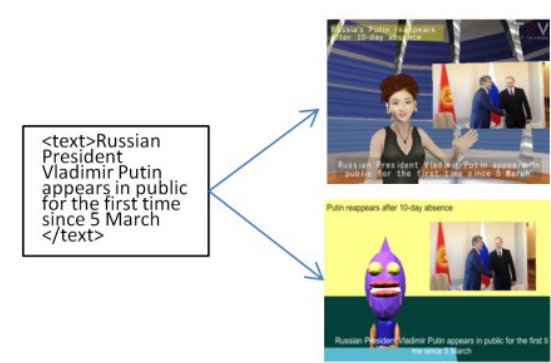

Figure 3: Different show formats applied to the same text

- To enable a user to choose her or his favorite visual presentation manner for the same story. (i.e. favorite announcer, favorite studio set, etc. see Figure 3)

- $\quad$ To process the story relatively easy (i.e. to translate to other languages using machine translation, to make a digest version of the original story, etc.)

On the other hand, drawback of the method would be that the quality of the output CGA would be affected by the fact of cutting a way of interaction between the story and the visual.

\section{ARCHITECTURE OF THE FRAMEWORK}

This chapter describes how we deploy the method actually in our framework.

Figure 4 shows the architecture. As explained in the previous chapter, SEC and ARC are text processing engines. We deploy those engines on the server as PHP programs (obviously, it does not necessarily have to be PHP, it could be Perl, Python, etc.) where a client side can utilize those functions via network. The SEC engine works as CRON on the server accessing the target source Web pages periodically to update the ARC script so that the ARC script is maintaining the latest story. And the ARC module is also on the server as PHP program file which is ready to receive a request from the client for the conversion from the ARC script to TVML script. Then ultimately the TVML script is played back by a TVML engine at the client side and the user can watch the CGA. The PHP codes of SEC and ARC can be uploaded and registered to the server by any individual by using a service page on the Web.

\section{THE ENGINES}

This chapter describes the detail of three engines: SEC engine, ARC engine and TVML engine.

\section{A. SEC engine}

As explained before, the SEC engine converts HTML to ARC script. Apparently, the design of the engine depends on a structure of a target Web page. If the target HTML is changed, the engine needs to be updated to be able to analyze it, otherwise it cannot produce an ARC script correctly. But if the HTML is standardized following a rule of, for example, Semantic Web, the engine should work despite the revision of the target page. And if we use RSS data for the analysis, it would work right owing to the RSS standardization.

Furthermore, if the target page is a personal Blog using Content Management System (i.e. Wordpress), it is feasible to 


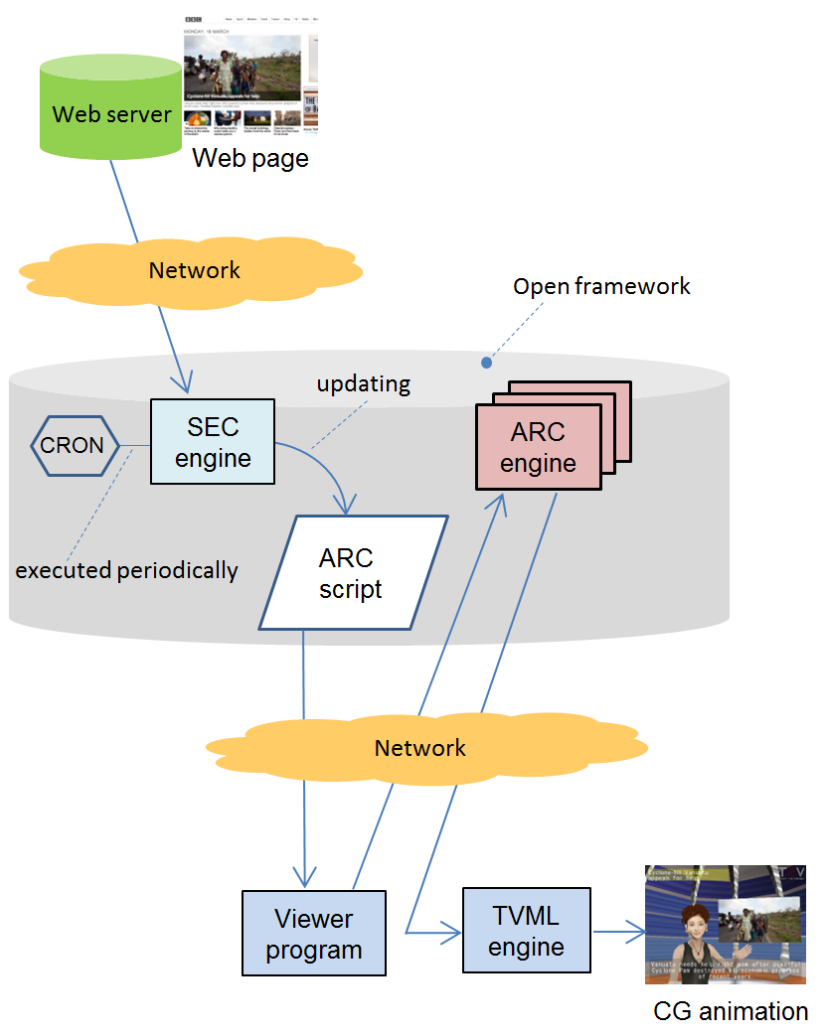

Figure 4: Architecture of the framework

give the engine consistency. And apparently, if the same person or organization maintain the target page and the ARC engine, it would be easy to keep up the simultaneous update for the both. Our solution for this matter is simple. We will leave it up to the developers by providing them the open framework where they can maintain their services in a consistent way.

\section{B. ARC engine}

The ARC engine converts ARC script to TVML script. Designing matter of this engine is first to make a specification of ARC script written in XML. We do not fix the strict specification for this. Instead, we open it to the developers. However, we have a basic standard specification of ARC script and also provide an ARC engine capable of the conversion. Table 1 shows some excerpt from the standard ARC specification. The standard ARC engine is made using PHP code and is on the server and ready to use. The usage of the ARC is simple. A client program passes the ARC script text data by POST request together with a variable indicating the name of the ARC engine, then the data is processed in the server executing the PHP program and return the result text to the client.

\section{TVML engine}

TVML is a special language to describe TV-program-like CGA. Table 2 shows visual and audio effects available in TVML. It mostly covers the functions necessary for producing TV-program-like animation. The TVML engine to interpret the TVML has been developed for years and a Windows version and a Unity version are now available. This time, we use the
Table 1: Standard ARC specification (excerpt)

\begin{tabular}{|l|l|l|}
\hline$<$ character $>$ & Mia, Bob, Mary,... & $\begin{array}{l}\text { which character is } \\
\text { assigned }\end{array}$ \\
\hline$<$ set $>$ & office, news show,... & which set is used \\
\hline$<$ text $>$ & text & $\begin{array}{l}\text { Character speaks the } \\
\text { text }\end{array}$ \\
\hline$<$ Subimage $>$ & URL of an image & $\begin{array}{l}\text { Display the image in } \\
\text { the set }\end{array}$ \\
\hline$<$ <action $>$ & bow, point out, $\ldots$ & Character action \\
\hline$<$ <article $>$ & text & $\begin{array}{l}\text { Superimpose the } \\
\text { text at a left corner }\end{array}$ \\
\hline$<$ pause $>$ & indicate number in & $\begin{array}{l}\text { Insert pause for a } \\
\text { specified time }\end{array}$ \\
\hline
\end{tabular}

Unity version of TVML SDK (System Development Kit). TVML engine works combining the input script with various necessary data such as character and studio CG modeling data, image, sound and movie data, etc. The engine can refer those data both on a local machine and on a server by specifying URL.

And owing to the Unity multiplatform ability, the engine can work on various types of platform such as PC, smart phone, game machine and even on a Web browser using "Unity Web Player".

\section{DEMONSTRATIONS}

We have developed the open framework explained in the previous chapters and produced substantial contents working on the framework. In this chapter, we introduce some attempts that we have done with it.

\section{A) Automatic News Show}

We have tried several different News sites such as Reuters, Google News, BBC, etc. Among those, here we introduce the automatic News Show using RSS data of "BBC World News". SEC engine is designed to extract RSS tags and to make an ARC script. Also we have designed ARC engine to simulate an existing News show format. Figure 5 shows the output. The

Table 2: TV production versus TVML event type

\begin{tabular}{|l|l|l|}
\hline \multicolumn{1}{|c|}{ Category } & \multicolumn{1}{|c|}{ TV production } & \multicolumn{1}{c|}{ TVML } \\
\hline Studio shot & Studio set & set \\
\cline { 2 - 3 } & Prop & prop \\
\cline { 2 - 3 } & Actor & character \\
\cline { 2 - 3 } & Lighting & light \\
\cline { 2 - 3 } & Camera & camera \\
\hline Footage & Video playback & movie \\
\hline Telop & Text, image display & title \\
\hline Superimpose & Text, image display & super \\
\hline Art work & Making figure & drawing \\
\hline Sound & Sound effect & sound \\
\cline { 2 - 3 } & Audio mixing & sound \\
\hline
\end{tabular}




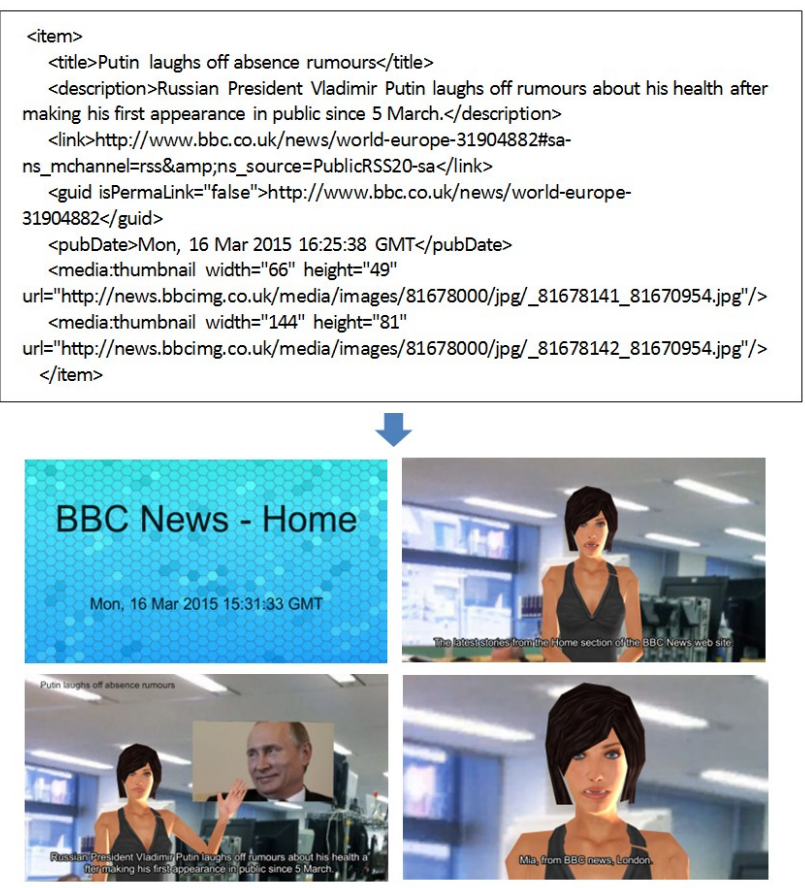

Figure 5: Automatic conversion from RSS of BBC News

SEC updates the ARC script once an hour, and a user can watch the latest news by accessing it. We have developed a client viewing software as PC application by using Unity.

\section{B) Automatic Talk Show}

We have made a SEC engine converting Japanese popular bulletin board (Ni channeru) to Talk Show. The SEC engine extracts speech text of each attendant and assigns the text to six characters randomly, then generates the ARC script. Figure 6 shows the output screen shots. The ARC engine here is quite special. It gives appropriate camera switching and where each character is looking at and their gesture (nodding) automatically based on an algorithm that we developed. The algorithm is rule-based one with statistic data obtained by analyzing a real TV talk show. Owing to the algorithm, the output animation is something that is fun to watch like a real talk show on a TV.

\section{C) Personal Blog}

We use Wordpress for constructing an automatic CGA
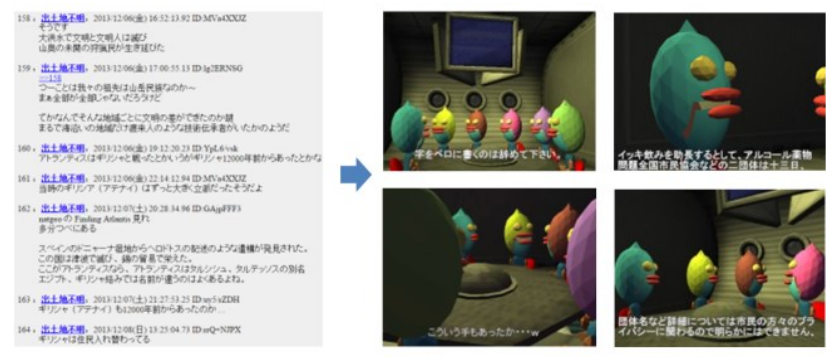

generation from a personal Blog in our framework. If the Blog uses a general Content Management System such as Wordpress, the HTML structure does not change unlike, for example, News sites driven by the establishments. So it would be easy to design the SEC engine. Here, we also set up a "hidden command" which activates the ARC engine to function as a certain control commands (i.e. to make a character move) but not appears on the Blog page. The Blog user inserts the command in the Blog text as a HTML comment (i.e. " $<$ !-(jump) -->" makes a character jump).

\section{CONCLUSION}

We have proposed and developed an open framework for automatic generation of $\mathrm{CG}$ animation from Web site. The HTML of a Web site is obtained by a server side program called "SEC" to produce ARC script in XML format. The ARC script is then processed by a visualization engine called "ARC" to produce TVML script. The TVML script is ultimately played back by a TVML engine at a client side. Using this framework, individual people enable to develop their own SECs and ARCs by PHP programming and to deploy them on the server so that people can access to watch the CGAs by special viewing software for PC. The viewer is developed by Unity game engine with TVML SDK. We have also developed several automatic CGA generation services on the framework such as automatic News Show, automatic Talk Show and personal Blog visualization. You can actually see the service site at http://niz237gt.sakura.ne.jp/budatv/

Our ultimate goal is to establish the automatic TV broadcast station on the Internet. We have successfully showed the potential of our concept by providing automatic CGA through our framework. In this paper, we introduced the outline of the entire framework, however, there are many details left in the development of the whole system which include: improvement of the service quality, security problem, content rights problem, implementation of a client side interactive service, and more. We are now challenging those aspects towards the future.

\section{REFERENCES}

[1] M. Hayashi, et al.: "T2V: New Technology of Converting Text to CG Animation", ITE Transactions on Media Technology and Applications, Vol.2, No.1, pp.74-82 (2014)

[2] M. Hayashi: "TVML (TV program Making Language) - Automatic TV Program Generation from Text-based Script -", In Proceedings of Imagina'99, pp.119-133 (1999)

[3] A. Nadamoto: "Complementing your TV-viewing by web content automatically-transformed into TV-program-type content", In Proceedings of the 13th annual ACM international conference on Multimedia, ACM. (2005)

[4] K. Tanaka: "Research on Fusion of the Web and TV Broadcasting. In Informatics Research for Development of Knowledge Society Infrastructure, 2007. ICKS 2007. Second International Conference on (pp. 129-136). IEEE. (2007)

[5] M. Hayashi, et al.: "Text Generated TV - A New Television System Delivering Visual Content Created Directly by Text -", In proceedings of IWAIT2014 (2014)

Figure 6: Automatic conversion from bulletin board 